\title{
CORRIGENDUM
}

\section{CORRIGENDUM TO: A FAST ALGORITHM FOR CALCULATING $S$-INVARIANTS}

\author{
DIRK SCHÜTZ \\ Department of Mathematical Sciences, Durham University \\ e-mail:dirk.schuetz@durham.ac.uk
}

(Received 28 July 2021; revised 11 August 2021; accepted 26 August 2021;

first published online 20 September 2021 )

Table 1 in [Sch21] claims to list all knots $K$ with up to 15 crossings for which one entry of $s^{\mathrm{Sq}^{1}}(K)$ differs from $s^{\mathbb{F}_{2}}(K)$. However, the table is incomplete. We list the missing knots in Table 1' below.

Table 1'. Prime knots with non-standard $s^{\mathrm{Sq}^{1}}$ missing from Table 1.

\begin{tabular}{lccr}
\hline Knot & $s^{\mathrm{Sq}^{1}}$ & $s^{\mathbb{F}_{2}}$ & $s^{\mathbb{F}_{3}}$ \\
\hline $15 n 154386$ & $(2,2,0,0)$ & 0 & 2 \\
$15 n 165952$ & $(2,2,0,0)$ & 0 & 2 \\
$15 n 165966$ & $(2,2,0,0)$ & 0 & 2 \\
$15 n 166064$ & $(2,2,0,0)$ & 0 & 2 \\
$15 n 166244$ & $(0,0,-2,-2)$ & 0 & -2 \\
\hline
\end{tabular}

The original computation was done in batches of 10,000 knots. It appears that only the first 150,000 non-alternating 15-crossing knots were checked. A subsequent computation confirmed the results in Table 1, but also found the knots in Table 1' among the remaining 18,030 non-alternating 15 -crossing knots.

\section{REFERENCE}

[Sch21] Dirk Schütz, A fast algorithm for calculating S-invariants, Glasg. Math. J. 63 (2021), no. 2, 378-399. MR 4244204 